\title{
Irinotecan Plus Gemcitabine and Fluorouracil in Advanced Biliary Tract Cancer: A Retrospective Study
}

\author{
Esther Endlicher ${ }^{\mathrm{a}} \quad$ Elisabeth Schnoy ${ }^{\mathrm{a}}$ Martina Troppmann ${ }^{\mathrm{a}}$ Gerhard Rogler $^{\mathrm{d}}$ \\ Helmut Messmann ${ }^{b}$ Frank Klebl ${ }^{a}$ Cornelia Gelbmann ${ }^{a}$ Frank Kullmann ${ }^{c}$ \\ aDepartment of Internal Medicine I, University Hospital Regensburg, Regensburg, ${ }^{b}$ Department of Internal Medicine \\ III, Klinikum Augsburg, Augsburg, and ' Department of Internal Medicine I, Klinikum Weiden, Weiden, Germany; \\ ${ }^{\mathrm{d}}$ Department of Internal Medicine, University Hospital Zurich, Zurich, Switzerland
}

\section{Key Words}

Biliary tract cancer - Chemotherapy - Irinotecan .

Gemcitabine · Fluorouracil

\begin{abstract}
Background: Since 2010, combination therapy with gemcitabine and cisplatin is the standard treatment for patients with biliary tract cancer (BTC) based on the ABC-02 trial. However, treatment after first-line progression is less clearly defined. We therefore retrospectively analyzed the efficacy of a 3-drug chemotherapy regimen in patients with advanced BTC. Methods: Patients with advanced BTC treated with palliative chemotherapy between April 2000 and October 2005 at Regensburg University Hospital were reviewed retrospectively. We analyzed the efficacy and safety of an institutional standard 3-drug regimen consisting of irinotecan, gemcitabine and 5-FU (IGF). One cycle, lasting 21 days, included applications on days 1 and 8 consisting of 75 $\mathrm{mg} / \mathrm{m}^{2}$ irinotecan i.v. for $90 \mathrm{~min}, 1,000 \mathrm{mg} / \mathrm{m}^{2}$ gemcitabine i.v. for $30 \mathrm{~min}$ and $2,000 \mathrm{mg} / \mathrm{m}^{2}$ fluorouracil (5-FU) for $24 \mathrm{~h}$. Results: A total of 12 histologically confirmed cases with gallbladder cancer and intrahepatic BTC were reviewed. Fifty percent of the patients $(6 / 12)$ had been pretreated with other chemotherapies. Median progression-free survival
\end{abstract}

was 9.4 months (1.5-21.5) and median overall survival was 17.2 months (2.5-24.3). Only neutropenia (8\%) was observed as an $\mathrm{NCl}-\mathrm{CTC}$ grade 3 toxicity. Anemia and leucopenia grades 1 and 2 were the most common side effects. Conclusions: The combination of IGF shows a promising survival benefit with manageable toxicity in patients with advanced BTC. Therefore, this regimen seems to be a feasible secondline treatment option for patients with rapid progression under first-line therapy with gemcitabine and cisplatin and with a good performance status.

ㄷ) 2016 S. Karger AG, Basel

\section{Introduction}

Cholangiocarcinoma are rare tumors with poor prognosis [1]. They originate intrahepatic or extrahepatic from the bile duct epithelium. Hilar carcinomas are known as Klatskin tumors. Gallbladder cancers are also associated with an unfavorable prognosis [2]. Surgery is the only curative treatment option with 5 -year survival rates of $0-40 \%$ after R0 resection [3-5]. In advanced,

E.E. and E.S. contributed equally.

\section{KARGER}

E-Mail karger@karger.com www.karger.com/dig
(C) 2016 S. Karger AG, Basel

0012-2823/16/0933-0229\$39.50/0
Esther Endlicher, MD

Department of Internal Medicine I

University of Regensburg

DE-93042 Regensburg (Germany)

E-Mail esther.endlicher@ klinik.uni-regensburg.de 
nonresectable tumors, the median overall survival (OS) for these patients is 3-6 months $[1,6,7]$.

Palliative therapy options are limited. Systemic chemotherapy with gemcitabine was the mainstay of treatment in advanced biliary tract cancer (BTC) for a long time. Median survival was about 6 months in different phase II studies [8].

In 2010, the first phase III randomized controlled multicenter trial (ABC-02) demonstrated a significant survival advantage for cisplatin plus gemcitabine compared with gemcitabine alone without an increase in toxicity [9]. In this study, 410 patients with locally advanced or metastatic BTC were randomly assigned to receive either cisplatin plus gemcitabine or gemcitabine alone. The median OS was 11.7 months in the combination group and 8.1 months in the gemcitabine group. Since this time, this combination therapy is the standard of care for the treatment of patients with advanced BTC.

A systematic review on gemcitabine and cisplatin for advanced BTC including one meta-analysis, 4 randomized controlled trials, 12 nonrandomized prospective studies and 3 retrospective studies supported the efficacy and safety of this combination [10]. Median OS ranged from 4.6 to 11.7 months without substantial toxicity.

Recently, a comprehensive analysis of 83 prospective trials in advanced BTC demonstrated that the combination of gemcitabine with platinum agents compared with other regimens shows a strong trend toward improved response rate, but no significant difference in progression-free survival (PFS) or OS [11]. Since the global effect of gemcitabine showed a strong statistical trend in OS and significant improvement in PFS while platinum containing regimens did not, this clinical effect seems mainly to be due to gemcitabine. Furthermore, in this study, gemcitabine-based combination therapies containing 5-FU showed a strong trend toward an improved OS (9.5 vs. 12.5 months) relative to platinum agents. To date, a randomized phase III trial comparing gemcitabine and cisplatin vs. gemcitabine plus 5 -FU is lacking.

Treatment protocols after first-line progression are less clearly defined. Depending on the first-line setting, 2 -drug combinations of gemcitabine and 5-FU or platin and 5-FU plus oxaliplatin or irinotecan are commonly used [12-14]. Irinotecan is a DNA topoisomerase I inhibitor, which has synergistic activity with 5-FU and gemcitabine [15]. Therefore, we retrospectively evaluate the efficacy of a palliative 3-drug chemotherapy protocol consisting of irinotecan, gemcitabine and 5-FU (IGF) in patients with nonresectable BTC.

\section{Patients and Methods}

\section{Patients}

We retrospectively analyzed individual chart reviews of 12 patients with histologically confirmed, nonresectable BTC, who were treated with a palliative chemotherapy consisting of IGF between April 2000 and October 2005 at the university clinic of Regensburg. Written consent was obtained in each case.

IGF regimen consisted of $75 \mathrm{mg} / \mathrm{m}^{2}$ irinotecan at a 90 - $\mathrm{min}$ infusion on days 1 and 8 , followed by $1,000 \mathrm{mg} / \mathrm{m}^{2}$ gemcitabine at a 30 -min infusion on days 1 and 8 and continuous infusion for $24 \mathrm{~h}$ of 2,000 $\mathrm{mg} / \mathrm{m}^{2} 5$-fluorouracil (days 1 and 8 ). This cycle was given every 21 days.

Tumor response was evaluated with CT scans usually performed every 2 cycles. Tumor response was evaluated and graded using WHO criteria [16]. A complete response (CR) per site was defined as the complete disappearance of the disease. A partial response (PR) was defined as a $>50 \%$ decrease in the total lesion size without appearance of new lesions. Progressive disease (PD) was characterized either as a $\geq 25 \%$ increase in lesion size or appearance of new lesions. Stable disease (SD) was defined as a CR, PR or PD not demonstrated $\geq 3$ weeks after starting treatment.

We excluded 2 patients from tumor response analysis: 1 patient received only one cycle of chemotherapy and was not available for response evaluation due to rapid progression. Another patient was not included because of a diagnosis of a second tumor (colorectal cancer) during chemotherapy.

\section{Statistical Analysis}

Response rate, PFS, OS and safety were measured. Patients who received at least one infusion were assessable for safety. For hematologic and biologic parameters, at least one measurement per cycle was required. OS was defined as the time from the beginning of chemotherapy until death. PFS was defined as the time from the beginning of chemotherapy until disease progression or death, whichever occurred first. The duration of response was calculated as the time from the beginning of chemotherapy to progression.

The Kaplan-Meier method was used to estimate OS and PFS outcomes. Statistical analysis was performed using the SPSS program (version 12.0 for Windows).

\section{Results}

\section{Patient Characteristics}

From April 2000 to October 2005, 12 patients were enrolled in the study. The baseline characteristics of the 12 treated patients are listed in table 1. Fifty-eight percent female and $42 \%$ male patients were treated. The median age was 69 (range 49-73 years). Seventy-five percent (9/12) of patients had intrahepatic cholangiocarcinoma; $25 \%(3 / 12)$ had gallbladder cancer.

Six of 12 patients (50\%) were pretreated with different chemotherapy protocols with pioglitazone, rofecoxib, capecitabine, gemcitabine and oxaliplatin (table 1). 


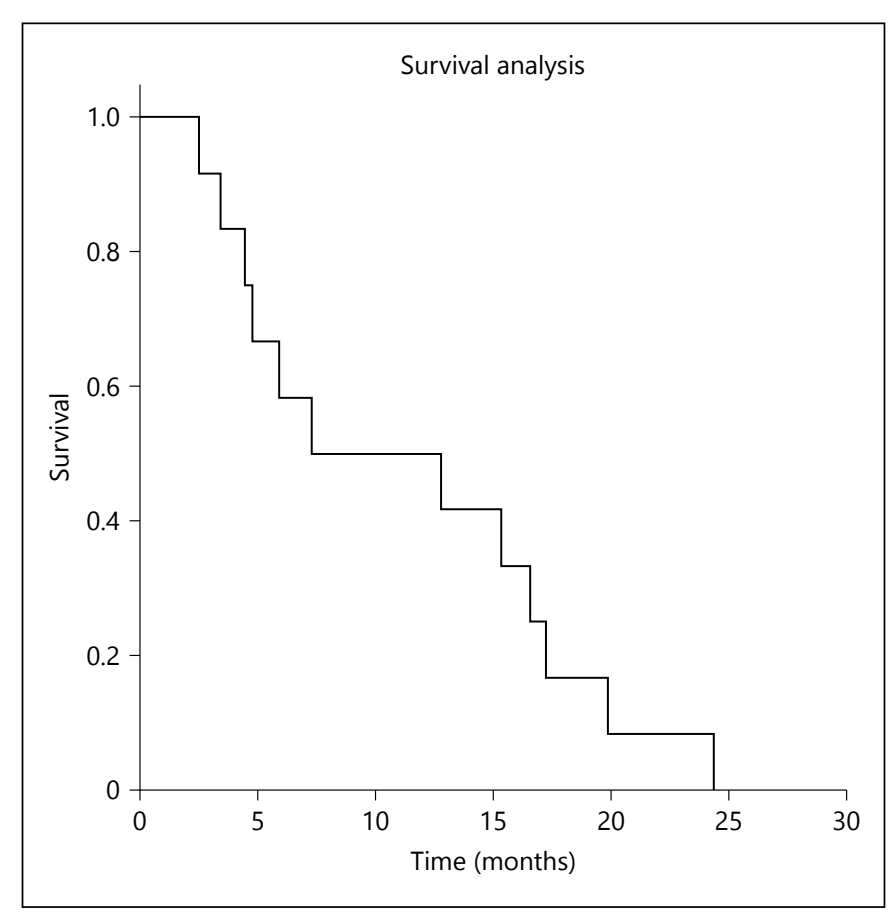

Fig. 1. Kaplan-Meier estimates of OS.

\section{Treatment Administration}

A total of 80 cycles were completed (median 5 cycles per patient; range 1-16 cycles). All patients received at least one dose of chemotherapy and were eligible for safety analysis. The median dose-intensity was $99 \%$ for irinotecan (50.7-101.8\%), $100 \%$ for gemcitabine (50.0$100.0 \%)$ and $100 \%$ for $5-\mathrm{FU}(49.5-100.5 \%)$.

\section{Toxicity}

Twelve patients could be assessed for safety. No treatment-related deaths occurred. NCI-CTC grades 3 and 4 toxicity were rare. Severe hematologic toxicities were observed only with respect to neutropenia (grade 3: 8\%). Anemia grades 1 and 2 were regarded as a common side effect of the chemotherapy $(8 / 12,67 \%$ and $3 / 12,25 \%$, respectively). Despite antiemetic medication, nausea affected most of the patients. Sixty-seven percent (8/12) of patients suffered from nausea. Grade 1 vomiting was observed in $33 \%(4 / 12)$ of patients and grade 2 in $17 \%(2 / 12)$ of patients. Three patients (25\%) experienced diarrhea grades 1 and 2 and only 1 patient (8\%) had grade 3 .

\section{Objective Response and Survival}

Ten patients could be assessed for response analysis (table 2; fig. 1). No objective treatment responses or regression of tumors were observed. Seven patients (70\%)
Table 1. Characteristics of treated patients

\begin{tabular}{lll}
\hline Number of patients & $\mathrm{n}=12$ & $\%$ \\
\hline Gender & 5 & 41.7 \\
$\quad$ Male & 7 & 58.3 \\
$\quad$ Female & 69 & - \\
Age, years & $49-73$ & - \\
$\quad$ Median & & \\
$\quad$ Range & 9 & 75 \\
Localization of the primary tumor & 3 & 25 \\
$\quad$ Intrahepatic cancer & & \\
$\quad$ Gallbladder cancer & 5 & 41.7 \\
Prior treatment & 1 & 8.3 \\
$\quad$ None & 6 & 50 \\
$\quad$ Operative resection & & \\
$\quad$ Chemotherapy* & &
\end{tabular}

* Different chemotherapy protocols with pioglitazone, rofecoxib, capecitabine, gemcitabine and oxaliplatin.

Table 2. Efficacy results

\section{Anzahl \%}

Tumor response after 6 cycles $(n=10)$

$\begin{array}{llr}\text { CR } & 0 & 0 \\ \text { PR } & 0 & 0 \\ \text { SD } & 7 & 70 \\ \text { PD } & 3 & 30\end{array}$

\begin{tabular}{lc}
\hline Time to tumor progression $(\mathrm{n}=12)$, months & \\
$\quad$ Median & 9.436 \\
Range & $1.51-21.53$ \\
OS $(\mathrm{n}=12)$, months & \\
Median & 17.24 \\
Range & $2.50-24.32$
\end{tabular}

had SD. For survival analysis all 12 patients were included: median PFS was 9.4 months (1.5-21.5); median OS was 17.2 months (2.5-24.3).

\section{Discussion}

BTC is a rare and aggressive tumor. The treatment of metastatic BTC still remains a challenge. In our retrospective analysis of patients with advanced BTC, we evaluated the efficacy and safety of a 3-drug regimen consisting of IGF. While the response rate was $0,70 \%$ of patients had SD with a promising median PFS of 9.4 months and a median OS of 17.2 months. There were no grade 4 tox- 
icities, and grade 3 side effects such as leucopenia were found only in 1 patient (8\%).

There are few published reports about the treatment of advanced BTC with 3-drug combinations and to our knowledge determined only in first-line treatment so far: Petrioli et al. [17] could show that 3-weekly oxaliplatin combined with gemcitabine and oral capecitabine in the first-line treatment of 37 patients with advanced BTC is an active and well-tolerated chemotherapy regimen. The overall response rate was $35 \%$ and the disease control rate was $73 \%$. The median PFS was 9.4 months (95\% CI 4.112.2 months) and the median OS was 13.8 months (95\% CI 7.7-17.1 months). Analogous to our study, there were no grade 4 toxicities and grade 3 neutropenia in $13.5 \%$ of patients and grade 3 thrombocytopenia in $10.8 \%$.

In a multi-institution phase II study, Kanai et al. [18] evaluated the efficacy and safety of gemcitabine/cisplatin/ S-1 combination chemotherapy in 50 patients with advanced BTC. Comparable to our data, the median OS was promising with 16.2 months. Toxicity was manageable with grades 3-4 neutropenia in 32\%, anemia in 32\% and thrombocytopenia in $10 \%$. Febrile neutropenia occurred in $4 \%$.

In 2010, Yamashita et al. [19] also reported a significant antitumor activity of gemcitabine/5-FU/cisplatin in
21 patients with advanced BTC: median OS was 18.8 months and PFS was 13.4 months. Hematological grade 3 toxicity, such as leucopenia, thrombocytopenia and anemia, was found in 6 patients $(28.6 \%)$.

A further small study on 14 patients with advanced BTC could demonstrate an overall response rate of $21.4 \%$ with a triple combination chemotherapy consisting of gemcitabine, 5-FU and cisplatin [20]. SD was observed in $64.3 \%$ and clinical benefit in even $85.7 \%$.

On the other hand, Wagner et al. [21] achieved comparable results for response and survival to previously reported regimens (median OS 10 months) with the combination of gemcitabine, oxaliplatin and 5-FU in patients with advanced BTC and gallbladder cancer, but with more toxicity.

However, according to others (17-20), a 3-drug combination chemotherapy was a safe and feasible treatment option in the second-line setting, which resulted in a clinically relevant stabilization rate in the pretreated patients with advanced BTC. Consequently, IGF might be a helpful treatment option for patients with BTC and a good performance status after rapid progression under first-line therapy with gemcitabine and cisplatin.

\section{References}

1 Khan SA, Thomas HC, Davidson BR, TaylorRobinson SD: Cholangiocarcinoma. Lancet 2005;366:1303-1314.

2 Misra S, Chaturvedi A, Misra NC, Sharma ID: Carcinoma of the gallbladder. Lancet Oncol 2003;4:167-176.

3 DeOliveira ML, Cunningham SC, Cameron JL, Kamangar F, Winter JM, Lillemoe KD, Choti MA, Yeo CJ, Schulick RD: Cholangiocarcinoma: thirty-one-year experience with 564 patients at a single institution. Ann Surg 2007;245:755-762.

4 Ramacciato G, Nigri G, Bellagamba R, Petrucciani N, Ravaioli M, Cescon M, Del Gaudio M, Ercolani G, Di Benedetto F, Cautero N, Quintini C, Cucchetti A, Lauro A, Miller C, Pinna AD: Univariate and multivariate analysis of prognostic factors in the surgical treatment of hilar cholangiocarcinoma. Am Surg 2010;76:1260-1268.

5 Nuzzo G, Giuliante F, Ardito F, De Rose AM, Vellone M, Clemente G, Chiarla C, Giovannini I: Intrahepatic cholangiocarcinoma: prognostic factors after liver resection. Updates Surg 2010;62:11-19.

6 Farley DR, Weaver AL, Nagorney DM: 'Natural history' of unresected cholangiocarcinoma: patient outcome after noncurative intervention. Mayo Clin Proc 1995;70:425-429.
7 Chang WH, Kortan P, Haber GB: Outcome in patients with bifurcation tumors who undergo unilateral versus bilateral hepatic duct drainage. Gastrointest Endosc 1998;47:354-362.

8 Eckel F, Schmid RM: Chemotherapy in advanced biliary tract carcinoma: a pooled analysis of clinical trials. Br J Cancer 2007;96:896902.

9 Valle J, Wasan H, Palmer DH, Cunningham D, Anthoney A, Maraveyas A, Madhusudan S, Iveson T, Hughes S, Pereira SP, Roughton M, Bridgewater J; ABC-02 Trial Investigators: Cisplatin plus gemcitabine versus gemcitabine for biliary tract cancer. N Engl J Med 2010;362:1273-1281.

10 Park JO, Oh DY, Hsu C, Chen JS, Chen LT, Orlando M, Kim JS, Lim HY: Gemcitabine plus cisplatin for advanced biliary tract cancer: a systematic review. Cancer Res Treat 2015;47:343-361.

11 Ulahannan SV, Rahma OE, Duffy AG, Makarova-Rusher OV, Kurtoglu M, Liewehr DJ, Steinberg SM, Greten TF: Identification of active chemotherapy regimens in advanced biliary tract carcinoma: a review of chemotherapy trials in the past two decades. Hepat Oncol 2015;2:39-50.

12 Rogers JE, Law L, Nguyen VD, Qiao W, Javle MM, Kaseb A, Shroff RT: Second-line sys- temic treatment for advanced cholangiocarcinoma. J Gastrointest Oncol 2014;5:408413.

13 Feisthammel J, Schoppmeyer K, Mössner J, Schulze M, Caca K, Wiedmann M: Irinotecan with 5-FU/FA in advanced biliary tract adenocarcinomas: a multicenter phase II trial. Am J Clin Oncol 2007;30:319-324.

14 Chung MJ, Kim YJ, Park JY, Bang S, Song SY, Chung JB, Park SW: Prospective phase II trial of gemcitabine in combination with irinotecan as first-line chemotherapy in patients with advanced biliary tract cancer. Chemotherapy 2011;57:236-243.

15 Bahadori HR, Rocha Lima CM, Green MR, Safa AR: Synergistic effect of gemcitabine and irinotecan (CPT-11) on breast and small cell lung cancer cell lines. Anticancer Res 1999;19: 5423-5428.

16 Miller AB, Hoogstraten B, Staquet M, Winkler A: Reporting results of cancer treatment. Cancer 1981;47:207-214.

17 Petrioli R, Roviello G, Fiaschi AI, Laera L, Roviello F, Marrelli D, Francini E: Threeweekly oxaliplatin combined with gemcitabine and capecitabine in the first-line treatment of patients with advanced biliary tract cancer. Anticancer Drugs 2015;26:682686 
18 Kanai M, Hatano E, Kobayashi S, Fujiwara Y, Marubashi S, Miyamoto A, Shiomi H, Kubo S, Ikuta S, Yanagimoto H, Terajima H, Ikoma H, Sakai D, Kodama Y, Seo S, Morita S, Ajiki $\mathrm{T}$, Nagano $\mathrm{H}$, Ioka $\mathrm{T}$ : A multi-institution phase II study of gemcitabine/cisplatin/S-1 (GCS) combination chemotherapy for patients with advanced biliary tract cancer (KHBO 1002). Cancer Chemother Pharmacol 2015; 75:293-300
19 Yamashita Y, Taketomi A, Itoh S, Harimoto N, Tsujita E, Sugimachi K, Gion T, Maehara Y: Phase II trial of gemcitabine combined with 5-fluorouracil and cisplatin (GFP) chemotherapy in patients with advanced biliary tree cancers. Jpn J Clin Oncol 2010;40:2428.

20 Morine Y, Shimada M, Ikegami T, Imura S, Kanemura H, Arakawa Y, Hanaoka J, Kanamoto M, Nii A: Usefulness of gemcitabine combined with 5-fluorouracil and cisplatin (GFP) in patients for unresectable biliary carcinoma. Hepatogastroenterology 2009;56: 307-312.
21 Wagner AD, Buechner-Steudel P, Moehler M, Schmalenberg H, Behrens R, Fahlke J, Wein A, Behl S, Kuss O, Kleber G, Fleig WE: Gemcitabine, oxaliplatin and 5-FU in advanced bile duct and gallbladder carcinoma: two parallel, multicentre phase-II trials. Br J Cancer 2009;101:1846-1852. 